\title{
INHALTSVERZEICHNIS
}

\section{Einleitung -9}

\section{Grundlagen der Siedlungsforschung - 11}

2.1 Allgemeine Begriffe - 11

2.2 Der Naturraum - 12

\section{Frühmittelalterliche Siedlungsreste aus Niederösterreich - 13}

3.1 Angern, SG Herzogenburg,VB St. Pölten - 16

3.2 Baumgarten an der March, Gem. Weiden an der March,VB Gänserndorf - 18

3.2.1 Pflanzenreste aus Baumgarten/March (Ursula Thanheiser) - 21

3.2.2 Die Befunde - 21

3.2.3 Katalog -28

3.3 Bernhardsthal, MG Bernhardsthal,VB Mistelbach - 31

3.4 Bisamberg, MG Bisamberg,VB Korneuburg - 33

3.5 Eggenburg, SG Eggenburg,VB Horn - 34

3.6 Eggendorf am Wagram, MG Stetteldorf am Wagram,VB Korneuburg - 36

3.7 Falkenstein, MG Falkenstein,VB Mistelbach - 38

3.8 Franzhausen, MG Nußdorf ob der Traisen,VB St. Pölten - 38

3.9 Fugnitz, SG Geras, VB Horn - 40

3.10 Großsierning, Gem. Haunoldstein,VB St. Pölten - 41

3.11 Hohenau, MG Hohenau an der March,VB Gänserndorf - 41

3.12 Hollabrunn, SG Hollabrunn,VB Hollabrunn - 44

3.13 Hollenstein, MG Ziersdorf,VB Hollabrunn - 45

3.14 Inzersdorf an der Traisen, Gem. Inzersdorf-Getzersdorf,VB St. Pölten - 47

3.15 Mannersdorf am Leithagebirge, SG Mannersdorf am Leithagebirge,VB Bruck an der Leitha - 48

3.16 Mannersdorf, MG Angern an der March,VB Gänserndorf - 50

3.16.1 Die Befunde -52

3.16.2 Katalog -55

3.17 Michelstetten, MG Asparn an der Zaya,VB Mistelbach - 56

3.17.1 Zwischenbericht der archäobotanischen Auswertung (Marianne Kohler-Schneider und Silvia Wiesinger) - 57

3.17.2 Die Befunde - 59

3.17.3 Katalog -66

3.18 Mitterretzbach, Gem. Retzbach,VB Hollabrunn - 72

3.19 Neudorf, MG Neudorf bei Staatz,VB Mistelbach - 73

3.20 Neunkirchen, SG Neunkirchen,VB Neunkirchen - 75

3.21 Niederhollabrunn, MG Niederhollabrunn,VB Korneuburg - 76

3.21.1 Der Befund - 78

3.22 Ossarn, SG Herzogenburg,VB St. Pölten - 78

3.23 Ottenthal, Gem. Ottenthal,VB Mistelbach - 79

3.24 Pitten, MG Pitten,VB Neunkirchen - 80 
3.25 Poigen, Gem. St. Bernhard-Frauenhofen,VB Horn - 82

3.26 Reichersdorf, MG Nußdorf ob der Traisen,VB St. Pölten - 83

3.27 Rosenburg, Gem. Rosenburg-Mold,VB Horn - 86

3.28 Schönkirchen, MG Schönkirchen-Reyersdorf,VB Gänserndorf - 86

3.29 Sommerein, MG Sommerein,VB Bruck an der Leitha - 88

3.30 Stratzing, MG Stratzing,VB Krems - 88

3.31 Tulln-Südumfahrung, SG Tulln an der Donau,VB Tulln - 90

3.31.1 Tierknochen von Tulln-Südumfahrung (Christian Reisinger) - 92

3.31.2 Die Befunde - 95

3.31.3 Katalog -96

3.32 Unterradlberg, SG St. Pölten,VB St. Pölten - 98

3.33 Unterrohrbach, MG Leobendorf,VB Korneuburg - 101

3.34 Wagendorf, MG Sieghartskirchen,VB Tulln - 101

3.35 Wagram an der Traisen, SG Traismauer,VB St. Pölten - 103

3.36 Zwingendorf, MG Großharras,VB Mistelbach - 104

\section{Einzelne Siedlungsobjekte -107}

4.1 Grubenhäuser - 109

4.1.1 Heizeinrichtungen in Grubenhäusern - 114

4.2 Längliche Gruben mit muldenförmigem Querschnitt - Erdmieten - 115

4.3 Ebenerdige Bauten -117

4.4 Speichergruben -118

4.5 Brunnen -120

4.6 Teererzeugungsgrube -120

4.7 Sonstige Gruben -120

\section{Räumliche Strukturen innerhalb einer Siedlung - 121}

5.1 Forschungsgeschichtlicher Abriss - 121

5.2 Problematik der frühmittelalterlichen Chronologie - 122

5.2.1 Ältere Chronologieschemata aufgrund von Formkriterien - 123

5.2.2 Neuere Chronologieschemata - 125

5.2.3 Datierung der neu vorgelegten frühmittelalterlichen Siedlungsreste anhand gängiger Chronologieschemata -128

5.3 Räumliche Strukturen innerhalb der Siedlungen aus Niederösterreich - 129

5.3.1 Wirtschaftsformen und Siedlungsdauer im Frühmittelalter - 132

\section{Räumliche Strukturen innerhalb einer Siedlungskammer - 135}

6.1 Frühmittelalterliche Zentralorte in Niederösterreich - 135

6.1.1 Großer Buchberg bei Alland - 136

6.1.2 Bad Deutsch Altenburg - Kirchberg - 136

6.1.3 Böheimkirchen - Hochfeld - 136

6.1.4 Schanze bei Gars - Thunau - 137

6.1.5 Gloggnitz - Schafkogel - 138

6.1.6 Hardegg - 138

6.1.7 Heidenstatt bei Limberg - 138

6.1.8 Michelstetten - Steinmandl - 139

6.1.9 Oberleiserberg -139

6.1.10 Pitten - Schlossberg - 139

6.1.11 Oberndorf bei Raabs - Burg Raabs - 140

6.1.12 Oberpfaffendorf - Sand - 140

6.1.13 Burgstall bei Schiltern - 140

6.1.14 Senftenberg - Schanzenriedl - 141 
6.1.15 Stein - Altenburg - 141

6.1.16 Wieselburg - Kirchberg - 141

6.1.17 Ungesicherte frühmittelalterliche Zentralorte - 142

6.1.18 Irrtümlich als frühmittelalterlich bezeichnete Anlagen - 142

6.2 Siedlungen in ehemaligen römischen Anlagen - 144

6.2.1 Carnuntum - Bad Deutsch Altenburg - 144

6.2.2 Klosterneuburg -144

6.2.3 Mautern - Favianis - 145

6.2.4 Tulln-Comagena - 146

6.2.5 Zeiselmauer - 146

6.2.6 Zwentendorf - 146

6.3 Die Straßen Niederösterreichs im Frühmittelalter - 147

6.4 Frühmittelalterliche Siedlungskammern in Niederösterreich - 149

7. Zusammenfassung -155

8. Literatur -157

Abkürzungsverzeichnisse -166

Quellennachweis - 166

Tafeln - 167

\section{Günther Karl Kunst}

Tierreste aus der frühmittelalterlichen Siedlung von Baumgarten an der March, Niederösterreich - 197 
